\title{
Extensión de la escolaridad y obligatoriedad de la escuela secundaria en Argentina: el papel de la universalización de la lectura y escritura $^{1}$
}

\author{
Luisa Iñigo ${ }^{2}$ \\ Universidad de Buenos Aires, Argentina \\ inigoluisa@gmail.com \\ Victoria Rio ${ }^{3}$ \\ Universidad de Buenos Aires, Argentina \\ riovictoria@gmail.com \\ Recibido: 5 de febrero de 2016 \\ Aceptado: 31 de mayo de 2016 \\ Disponible en linea: 20 de dicembre de 2016
}

Artículo de revisión.

2 Socióloga, Universidad de Buenos Aires (UBA) (Argentina). Magíster en Generación y Análisis de Información Estadística, UNTREF (Argentina). Docente, UBA. Co-directora del proyecto UBACYT "El desarrollo de la acumulación de capital en la Argentina desde la década de 1960 a la actualidad: estructura económica y formas políticas" (Buenos Aires, Argentina)

3 Licenciada en Ciencias de la Educación, UBA. Maestranda en Ciencias Sociales del Trabajo, UBA. Docente, UBA. Integrante del proyecto UBACYT "Los ciclos de reformas educativas: cambios académicos y en la organización del gobierno del sistema educativo argentino. Período 1992-2010” (Buenos Aires, Argentina). 


\title{
Extensión de la escolaridad y obligatoriedad de la escuela secundaria en Argentina: el papel de la universalización de la lectura y escritura
}

\section{Resumen}

El presente artículo se propone abordar el vínculo entre la extensión de la escolaridad en Argentina y el desarrollo de la lectura y la escritura. Con ese fin, se parte de examinar el fenómeno de la universalización de esas capacidades en la sociedad capitalista, para luego analizar las transformaciones recientes en los procesos de trabajo que subyacen a esa extensión. Finalmente, se estudia el caso argentino en particular, en cuanto al papel de la escolarización de nivel secundario en el desarrollo de capacidades lectoras y escritoras. Para ello, se analizan los contenidos básicos curriculares del área de Lengua para el nivel secundario, así como algunos resultados de los Operativos Nacionales de Evaluación.

Palabras clave: lectura y escritura; extensión de la escolaridad; escuela secundaria; Argentina

\section{Schooling Extension and Compulsory Secondary Education in Argentina: the role of the Universalization of Reading and Writing Abilities}

\begin{abstract}
This article approaches the relation between extending compulsory schooling and the development of reading and writing abilities in Argentina. First, it examines the universalization of these abilities in the capitalist society, and then it analyzes recent transformations in labor processes that underlie the extension of the average schooling years. Finally, it focuses on the Argentinean case, specifically on the role of secondary schooling in the development of reading and writing abilities. To this end, both secondary school basic curricular contents in Language and the results of the "Operativo Nacional de Evaluación" (national assessment census) regarding this subject are analyzed.
\end{abstract}

Keywords: reading and writing; schooling extension; secondary school; Argentina

\section{Extensão da escolaridade e obrigatoriedade do ensino médio na Argentina: papel da universalização da leitura e escrita}

\section{Resumo}

O presente artigo propõe abordar a ligação entre o alargamento da escolaridade na Argentina e o desenvolvimento da leitura e a escrita. Com esse objetivo, examina-se o fenômeno da universalização de tais capacidades na sociedade capitalista, para analisar após as transformações recentes nos processos de trabalho que subjazem a tal extensão. Por fim, o caso argentino em particular é estudado, em relação ao papel da escolarização de nível secundário no desenvolvimento de capacidades de leitura e escrita. Para isso, são analisados os conteúdos básicos curriculares da área de Língua no ensino médio, bem como alguns resultados dos Operativos Nacionais de Avaliação.

Palavras-chave: leitura e escrita; extensão da escolaridade; ensino médio; Argentina 


\section{Introducción}

En Argentina, la pregunta por la extensión de la escolaridad ha adquirido centralidad durante las últimas décadas a partir de la expansión de la escuela secundaria y los recientes procesos de reforma educativa. En este sentido, en la década de 1990, la Ley Federal de Educación extendió la obligatoriedad a diez años, y en la década pasada la Ley de Educación Nacional sumó tres años más, estableciendo la obligatoriedad del secundario.

Este proceso, que coexiste con una tendencia mundial con el mismo sentido, ha suscitado y reeditado numerosas discusiones académicas. Algunas explicaciones sostienen, en consonancia con los desarrollos de Boudon (1983) y Bourdieu (1978, 1991), que la universalización del nivel primario fuerza, a su vez, una extensión de la carrera escolar media, al hacer que las titulaciones pierdan su valor relativo (Tenti, 1995; Filmus, 1998). Este planteo agrega que, en Argentina, aquella tendencia hacia la universalización habria tenido lugar mediada por la segmentación (Bravslavsky, 1989; Tiramonti, 1993) de las ofertas educativas en circuitos diferenciados. Así mismo, en los últimos años han surgido trabajos que explican la extensión de la obligatoriedad en Argentina como forma de la burguesía de extender su función ideológica de disciplinamiento de los trabajadores y "contención" de la creciente población obrera sobrante (De Luca, 2004).

Si bien dan cuenta de aspectos distintos del problema, estas explicaciones no llegan a responder la pregunta sobre la necesidad social general de la extensión de la escolaridad. En efecto, cabe preguntarse si la presión por una escolarización más extensa, la devaluación de las credenciales, la segmentación educativa y la función ideológica del Estado no pueden considerarse expresiones de un único contenido material.

El presente trabajo se propone contribuir a una explicación materialista de la extensión de la obligatoriedad escolar, a partir de retomar la pregunta por el papel de la universalización de la escolarización en la sociedad capitalista y la necesidad de la generalización 
de la lectoescritura. Según procuraremos demostrar, este camino, menos explorado por la literatura especializada, contiene elementos para avanzar en el conocimiento de las determinaciones más fundamentales y sustantivas del proceso de prolongación de la escolaridad y de su expresión legal en el establecimiento de la obligatoriedad de la escolaridad secundaria.

Con ese fin, en primer lugar, nos preguntaremos qué determinaciones generales de la sociedad capitalista subyacen a la universalización de la lectura y escritura a partir del siglo XIX. En segundo lugar, nos enfocaremos en las transformaciones en la materialidad del trabajo durante las últimas décadas del siglo XX, que podrian estar en el núcleo de la necesidad social por una extensión del periodo de aprendizaje formal de la lectura y escritura para, a continuación, establecer en qué medida esas transformaciones han tenido una expresión en el recorte nacional argentino de la acumulación mundial.

Finalmente, analizaremos las formas históricas de la extensión de la escolaridad en Argentina a partir de la década de 1980, para luego hipotetizar acerca del papel que el aprendizaje de la lectura y escritura ha jugado en ella. Para esto último, nos detendremos en el análisis de los resultados de las pruebas del Operativo Nacional de Evaluación (ONE) de la calidad educativa a partir de 2007 (DiNIECE, 2009, 2013), con el propósito de describir el contenido del pasaje por la educación secundaria en lo que se refiere al desarrollo de capacidades lectoras $-\mathrm{y}$, en menor medida, de escritura-, para diversos grupos de alumnos del nivel en la Argentina. 


\section{La lectura y escritura y el papel de su alcance universal en la sociedad capitalista}

\section{La lectura y escritura como desarrollo de las fuerzas productivas sociales}

El proceso de vida humano se funda en la transformación consciente del medio natural en un medio para la vida humana, es decir, se funda en el trabajo (Marx, 2008, pp. 215-216). Este proceso es, ante todo, de carácter social, donde "el gasto productivo del cuerpo de un individuo humano tiene como resultado la reproducción del cuerpo de otro individuo como ser humano" (Iñigo Carrera, 2008, p. 81). Por tanto, las fuerzas productivas de esta actividad son potencias del proceso de cooperación en tanto tal y existen bajo la forma de las subjetividades productivas individuales. En este punto, Iñigo Carrera (2008, p. 81) destaca el papel central que juega la comunicación en tal proceso:

La organización del proceso de metabolismo social toma así forma concreta en un proceso de comunicación recíproca mediante el cual los individuos coordinan sus trabajos individuales en la unidad del trabajo social, y este proceso no es sino la exteriorización recíproca del ser social de los individuos que se comunican entre sí.

Según Iñigo Carrera (2008, p. 82), en sus inicios esta exteriorización recíproca no pasa de la comunicación mediante gestos y sonidos,

Pero, en su desarrollo ya como un proceso organizado de manera consciente y voluntaria, el trabajo humano trasciende el alcance de cualquier sistema de signos que no pueda exteriorizar la ilación cada vez más compleja de las potencialidades de sujetos y objetos, organizadora de la acción conjunta. La coordinación de las subjetividades productivas individuales a través de la exteriorización del ser social de las mismas va tomando la forma concreta de un lenguaje, esto es, de un sistema de signos verbales que portan la unidad de los significados cada vez más complejos con que opera la conciencia. En sintesis, el lenguaje es la forma de relación social en que las 
subjetividades conscientes individuales coordinan su acción como órganos del proceso de metabolismo social.

Si la superación del mero signo por un sistema de signos verbales supone un salto crucial en el desarrollo de la capacidad productiva del trabajo social, otro salto semejante lo constituye la objetivación de ese sistema en un sistema de signos escritos. En palabras de Ong (1982), "la escritura, consignación de la palabra en el espacio, extiende la potencialidad del lenguaje casi ilimitadamente" (p. 17). Y por cierto que el hecho de dotar a la ilación consciente de una existencia objetiva, exterior a sus sujetos, porta la expansión del alcance de la relación social en el tiempo y el espacio en una escala desconocida hasta entonces.

Pero esta no es la única manera en que la escritura revoluciona las fuerzas productivas. En el mismo movimiento, libera al cerebro humano de la necesidad de almacenar el conocimiento social producido hasta el momento y, con ello, abre nuevos terrenos a su acción (Ong, 1982).

\section{La universalización de la lectura y escritura en la sociedad capitalista}

Tanto Ong (1982), como Hébrard (1988), Chartier y Hébrard (2000) y Chartier (2002) son enfáticos en presentar la lectura y la escritura como una relación social con proyección universal recién a partir de fines del siglo XIX ${ }^{4}$.

En la historia del modo de producción feudal, es solo entre los siglos VIII y XI que tiene lugar el pasaje de una cultura erudita oral, en la que lo escrito juega un papel secundario, a una cultura erudita

\footnotetext{
4 "La característica del siglo XIX fue crear instituciones para enmarcar la lectura de toda la población, intentando promover referencias compartidas por el conjunto del cuerpo social. Se trataba de textos laicos y no tanto los religiosos, como en los siglos XVII y XVIII. Por el contrario, el dominio de la escritura tenía, por entonces, ambiciones mucho más modestas: la escritura debía acompañar los aprendizajes escolares y dar las competencias necesarias para escribir en la vida privada (correspondencia familiar). Así, el hecho de saber escribir, es decir, producir textos en el dominio público, quedó restringido a una élite social. La masificación de la enseñanza secundaria durante la segunda mitad del siglo XX, tuvo como primer objetivo dar a una mayor cantidad de jóvenes, y más tarde a todas las nuevas generaciones, el poder de la escritura. Simultáneamente a este hecho, las necesidades sociales en materia de escritura aumentaron, tanto para usos profesionales como para la vida cotidiana" (Chartier y Hébrard, 2000).
} 
centrada en la escritura (Hébrard, 1988, p. 16). Hébrard habla de antecedentes más o menos aislados de escritura no erudita en los siglos XV y XVI en las ciudades mercantiles y, tanto como los otros autores referidos, señala una extensión progresiva de la capacidad de leer y escribir a lo largo de los cinco siglos que median entre aquel momento y el siglo XX. Solo en el curso histórico del modo de producción capitalista el lenguaje escrito se ha extendido hasta convertirse en una relación social de alcance universal ${ }^{5}$. Para poder conocer por qué esto es así, no pueden obviarse las preguntas sobre qué es el modo de producción capitalista y qué papel juega en el desarrollo de la subjetividad productiva del ser humano.

\section{El carácter privado del trabajo}

Lo que distingue a la organización capitalista de la producción social es el carácter privado e independiente que asume el proceso de trabajo (Marx, 2008, p. 89). Cuando el trabajo se realiza bajo una forma privada, la unidad general del proceso de producción y consumo solo puede establecerse a través del intercambio, esto es, dándole al producto del trabajo la forma fetichizada de mercancía. De modo que lo que se expresa como el valor o la intercambiabilidad de los productos del trabajo no es más que la interdependencia general que los individuos guardan entre sí (Marx, 2001, p. 91).

A medida que la producción de la vida humana pasa a estar organizada mediante el intercambio mercantil, las relaciones sociales que establecen los individuos van dejando de poder existir sin tomar forma en un soporte objetivo, independiente del encuentro personal directo. En consecuencia, la generalización de la producción de mercancías, con su necesaria expresión en la mediación dineraria de los intercambios, tiene la forma de una difusión del registro escrito y del cálculo algorítmico.

5 Esta afirmación no desconoce, sin embargo, que leer y escribir no constituyeron actividades idénticas en las diversas formaciones sociales en las que se leyó y se escribió (Chartier y Hébrard, 2000). La universalización de la lectura y escritura implica la generalización de formas particulares de leer y escribir. 


\section{La conciencia del productor de mercancias}

En el modo de producción capitalista la interdependencia social se presenta a los individuos libres como la intercambiabilidad de sus mercancías. Se carece de dominio sobre el carácter social de la propia actividad y, por lo mismo, no es posible actuar de manera directa en calidad de órgano individual del trabajo social.

En ausencia de relaciones de dominio directo que regulen la acción individual, esta solo puede estar regida por la conciencia libre de los propios individuos. Puesto que no se trata de una conciencia libre en términos abstractos, la acción individual debe satisfacer a esa interdependencia en su unidad, por más libre que se considere a sí misma. La forma que asume el ejercicio del ser social en una sociedad de productores privados, y por lo tanto libres, consiste en que su interdependencia se presente a cada uno de ellos como una potencia exterior a la cual deben aprender a someterse, y a hacerlo voluntariamente (Iñigo Carrera, 2007, p. 59) .

Desde distintas perspectivas, se ha señalado que la enseñanza escolar ha sido forma privilegiada de la producción de esta conciencia por parte de los individuos ${ }^{7}$. Menos explorada, en cambio, ha sido la cuestión de si el aprendizaje de la lectura y escritura tiene algún papel específico en esa producción.

Si bien un tratamiento en profundidad del tema excedería el alcance de este artículo, desearíamos al menos dejar marcadas

6 Como afirmábamos en otro lugar, los productores de mercancía "no pueden 'descansar' sobre la posibilidad de verse forzados por otro a poner en movimiento su propia capacidad para el trabajo o a aplicarla de esta o aquella manera peculiar. Necesitan, por ende, desarrollar en sí la capacidad para ejercer autónomamente este dominio sobre sí mismos" (Hirsch e Iñigo, 2007, p. 8).

7 Para Althusser (1970/1988), el Estado se vale de sus aparatos ideológicos en la introyección de la ideología burguesa que supone el autorreconocimiento del individuo como sujeto. Esto constituye, para el autor, la condición de la reproducción de las relaciones sociales de producción capitalistas. La escuela es entonces el aparato ideológico por excelencia, dado su alcance universal y su pretensión de neutralidad. Bourdieu y Passeron (1979/1998) han descrito el papel fundamental de la escuela en la "reproducción cultural" de la sociedad capitalista a través del ejercicio de lo que denominaron "violencia simbólica". La autoridad pedagógica es la encargada de personificar y ejercer esta violencia a través de la cual se "impone" un sistema cultural arbitrariamente legítimo. Foucault (1976/2002), a su vez, describió las formas históricas que ha asumido la producción de esta capacidad de autosujetarse hasta el siglo XIX. En la obra referida el autor despliega de qué maneras la coacción fue dejando de ser el resultado de un "sobrepoder" personal y discontinuo del soberano con respecto a sus súbditos para convertirse en un proceso silencioso y permanente ejercido por cada cual sobre sí mismo (Hirsch e Iñigo, 2007). 
algunas líneas que encontramos sugerentes. Hébrard (1988) señala que hasta entrado el siglo XVII persiste en Europa una diferencia entre la catequización de católicos y protestantes: si para los primeros la fe "viene del oído" y lo escrito juega un papel solo para el sacerdote que la transmite, entre los protestantes la lectura es el vehículo necesario de satisfacción de "la exigencia de cotejo con el escrito doctrinal y espiritual que caracteriza a la devotio moderna" (Hébrard, 1988, p. 41). Cabe preguntarse si ese contraste no es expresivo de que la lectura y escritura y en especial la lectura -en cuanto habilitan el pensamiento interiorizado y la apropiación solitaria del conocimiento (Ong, 1982, pp. 77-78, 147-149) ${ }^{8}$ - constituyen una modalidad especialmente propicia para la producción de la conciencia individual que realiza el ser social, no bajo la forma de la subordinación personal directa, sino mediante la libre voluntad individual.

\section{La producción de plusvalia relativa y el desarrollo de la ciencia}

La mercancía solo se constituye en la relación social general cuando es el producto del capital. Por su parte, la producción de plusvalía relativa bajo la modalidad de la gran industria es la forma más potente y desarrollada en la que existe la producción mercantil. Al objetivarse los saberes y pericias manuales de los trabajadores en el sistema de maquinaria, se ven eliminadas las "trabas personales inherentes a la fuerza de trabajo humana" quedando subordinado, como nunca antes, el trabajo al capital (Marx, 2005). Pero el desarrollo de la gran industria requiere del sometimiento de "todos los aspectos de la producción al conocimiento científico" (Iñigo Carrera, 1993). Así, el desarrollo de la ciencia avanza sobre el control de la naturaleza, revolucionando de manera constante las fuerzas productivas sociales. Esta extracción siempre aumentada de plusvalía relativa mediante la aplicación de la ciencia al proceso productivo está portada en un fragmento de la clase obrera, cuyo trabajo es desarrollar la ciencia básica y sus aplicaciones tecnológicas, tanto como el control y la organización de la producción. Sin embargo, en tanto el proceso de trabajo

8 Ver también, al respecto, la referencia de Chartier y Hébrard (2000) a la progresiva expansión de la práctica de la lectura solitaria y silenciosa, que facilita la relación individual con el texto escrito por contraposición a la lectura recitada. 
es cada vez más el producto objetivado de ese desarrollo científico, el capital también necesita formar a la parte restante del colectivo de trabajadores - cuyos saberes y pericias son objetivados en la maquinaria- con atributos universales, apropiados a la interacción con los resultados de aquel desarrollo - v. gr., la comprensión de la "lógica" y los ritmos con los que funciona la máquina, la capacidad de cooperar con otros trabajadores al interior de la unidad de producción, etc.Esto, pese a que el desarrollo técnico "desespecializa" a este tipo de trabajador, al tiempo que tiende a eliminar la percepción sensorial y la habilidad manual de su trabajo9.

Por último, como toda la producción de valores de uso está sometida a ese desarrollo, en la medida en que este avanza, la participación en el proceso de consumo requiere cada vez más de la capacidad de emplear instrumentos portadores de ese conocimiento científico objetivado.

Puesto que el pensamiento lógico es la forma histórica actual de la conciencia científica (Iñigo Carrera, 1993), tanto la actividad de desarrollo científico y tecnológico como la interacción con sus productos requieren que los individuos desarrollen en sí la capacidad de pensar lógicamente. Vigotsky (1983) ha expuesto el papel del aprendizaje del lenguaje, y en particular del lenguaje escrito, en ese desarrollo: los resultados del aprendizaje de la capacidad para comunicarse por escrito no se limitan a lo "instrumental".

En particular, Vigotsky ha puesto énfasis en el papel del carácter de signos que puede darse a las palabras sobre la capacidad de pensar a través de conceptos. Así mismo, ha señalado que el aprendizaje del lenguaje escrito involucra un pensamiento de alto nivel de abstracción, la capacidad de distanciarse de las necesidades inmediatas, el desarrollo de una acción analítica y la realización de un trabajo consciente (Vigotsky, 1983). Las aptitudes que se desarrollan mediante el aprendizaje de la escritura son condiciones del ejercicio del pensamiento objetivable, en cuanto este "requiere lo que se ha llamado una

9 Cabe aclarar que no estamos haciendo referencia exclusiva al trabajo manufacturero directo sino también al desarrollo y organización del proceso productivo total (incluyendo el desarrollo de la ciencia básica y aplicada, los trabajos de la circulación y el trabajo de formación y reproducción de la fuerza de trabajo, por mencionar solo algunos). 
semántica deliberada, una estructuración intencional de la trama del significado" (Vigotsky, 1983, p. 138).

Resulta también elocuente la investigación de Luria (1980) sobre el desarrollo de la conciencia humana como producto de la historia social. El autor expuso cómo el dominio del lenguaje escrito está asociado a procesos cognitivos vinculados a la combinación de abstracción y generalización, la deducción, el razonamiento y la imaginación, frente a procesos psíquicos relacionados con los reflejos prácticos y concretos presentes en personas analfabetas, y cómo estos procesos están determinados por la materialidad de la vida social ${ }^{10}$.

La formación de atributos productivos universales, incluido el aprendizaje de la lectoescritura, se realiza, como es sabido, mediante el proceso de escolarización. No ha sido hasta el desarrollo del sistema de gran industria que comenzó a regularse el establecimiento de un periodo de escolaridad obligatorio universal -aunque su universalidad se haya concretado de manera progresiva- ${ }^{11}$. En los próximos apartados analizaremos las formas históricas que caracterizaron el desarrollo reciente de la gran industria para avanzar en la comprensión de las determinaciones de la extensión de la escolaridad a partir de mediados del siglo XX.

10 "Tal forma del pensamiento práctico concreto no es, sin embargo, innata ni predeterminada; es el resultado de unas formas elementales de práctica social que son las que dominan en este grupo de sujetos, es el resultado de su analfabetismo; la modificación de la práctica, la introducción de la gente en unas formas superiores de vida social, la asimilación de la lectura y la escritura, conducen a la creación de nuevos motivos que han sido provocados por formas más complejas de actividad, conducen a una rápida reconstrucción del pensamiento, a la adquisición de las operaciones teóricas y categoriales que antes se interpretaban como secundarias" (Luria, 1980, p. 112).

11 En la literatura existe acuerdo en que el desarrollo de la escuela moderna tiene por objetivo formar a los "ciudadanos y trabajadores" de la sociedad capitalista (Cuesta Fernández, 2005). Si bien excede los límites de este artículo, consideramos que esa distinción da por supuesto que la forma general de organización del trabajo social y su manifestación en un sistema de relaciones políticas guardan una relación de exterioridad recíproca. Aquí, en cambio, por atributos productivos entendemos a todos aquellos portados por los individuos en tanto órganos del trabajo social, tal como este se divide, realiza y gestiona en la sociedad capitalista (véase Hirsch e Iñigo, 2007). 


\section{Las transformaciones recientes en la materialidad del trabajo}

\section{Automatización y computarización de los procesos de trabajo}

Durante los últimos 50 años, la producción de plusvalía relativa a escala global ha asumido una forma específica, con consecuencias sobre el objeto de nuestro interés. La revolución de la materialidad del proceso de trabajo que tuvo lugar alrededor de la década de 1960 significó un avance sustancial en la mecanización de acciones que hasta alli formaban parte del trabajo manual directo, mediante la automatización y robotización a gran escala.

En el ámbito de la industria manufacturera tuvo lugar, de acuerdo con Balconi (2002), una segunda ola ${ }^{12}$ de "codificación de los saberes tácitos" de los trabajadores. Esta supuso la sustitución de competencias cognitivas específicas -adquiridas por los trabajadores calificados mediante la experiencia- por procedimientos e información codificados, a partir de "la creciente disponibilidad de instrumentos de medida y cálculo a bajo costo, los cuales proveyeron los datos cuantitativos a ser organizados en modelos y las capacidades computacionales para aplicar modelos y simulaciones" (Balconi, 2002, p. 360, traducción propia). La posibilidad de utilizar tecnologías de fabricación automatizadas fue el principal resultado de aquella sustitución.

Esto ha implicado transformaciones profundas en cuanto a los atributos productivos con que el capital demanda a los miembros del obrero colectivo. Más específicamente, ha involucrado una diferenciación interna que está en línea con la que caracteriza al desarrollo de la gran industria (Iñigo Carrera, 2008; Starosta, 2016;), al menos en tanto este no suponga la eliminación absoluta del trabajo directo.

Ha implicado, en primer término, una ulterior expansión de los atributos productivos de los asalariados a cargo de las porciones más complejas del proceso de trabajo. En segundo lugar, ha significado

\footnotetext{
12 Por referencia a la primera ola, taylorista, de la codificación (Balconi, 2002).
} 
un cambio en los tipos de capacidades que se requieren para realizar trabajo directo. Las habilidades tácitas de los trabajadores que se han vuelto así obsoletas son aquellas "que descansan en las percepciones de los órganos sensoriales y en la habilidad manual" (Balconi, 2002, p. 361, traducción propia), que requieren de la aplicación práctica y experiencia prolongadas, y solo marginalmente de la educación formal (Balconi, 2002, p. 362, traducción propia, resaltado nuestro). En cambio,

[1]as habilidades tácitas complementarias de los procesos productivos codificados y automatizados son aquéllas heurísticas e interpretativas que sirven para decodificar y dotar de significado a mensajes portadores de información (entradas de datos estructurados, procedimientos codificados) y para crear novedades ${ }^{13}$.

Estas presuponen una base de educación formal más o menos extensa -lo cual no significa que no se desarrollen ulteriormente mediante la experiencia- $\mathrm{y}$, al estar basadas en un conocimiento general, tienden a ser menos específicas a cada sector. De hecho, la movilidad intersectorial se ha visto facilitada por la reducción del tiempo y los costos de reentrenamiento específico (Balconi, 2002). En cuanto al foco de interés de este artículo, las tareas de control de los sistemas de máquinas automatizados suponen una apropiación fluida e inmediata de la palabra escrita, así como cierta habilidad para la expresión escrita ${ }^{14}$.

Estas transformaciones no parecen limitarse a los procesos de trabajo fabril que constituyen la referencia empírica de la bibliografia citada. La computarización de los procesos de trabajo excede claramente el ámbito de esa rama de la actividad económica.

${ }^{13}$ En un ejemplo que la autora toma de la industria de los semiconductores, "La computadora indica qué pruebas son necesarias antes de usar la máquina. En particular, los trabajadores deben analizar el gráfico de control y, si éste indica un problema, en la computadora aparece una lista de control que indica al trabajador que debe implementar una serie completa de verificaciones e intervenciones, al final de la cual se debe buscar ayuda si el problema no se resolvió. El manejo de toda la información provista por la máquina no es tarea trivial; ésta posee un servicio de autodiagnóstico, por lo que envía mensajes de advertencia más que de error. Las señales diagnósticas deben ser interpretadas, para decidir si seguir adelante o interrumpir la producción y pedir ayuda a un técnico" (Balconi, 2002, p. 368, traducción propia).

14 Todo lo antedicho no quita que, junto al controlador de la maquinaria, persista el trabajo manual — por ejemplo, de limpieza y traslado de objetos-, a cargo de trabajadores distintos de aquel. Lo que es más - y en tercer término-, en aquellos procesos no alcanzados por la automatización, se ha mantenido la demanda por los atributos "particularistas" antes referidos. 
La automatización y consiguiente diferenciación de los trabajadores de acuerdo con las líneas señaladas en el apartado anterior están en la base del nacimiento de la "nueva división internacional del trabajo", implicada por el desplazamiento de la porción del obrero colectivo encargada de la realización del trabajo más simple hacia países específicos del llamado "Tercer Mundo" (Fröbel, Heinrichs y Kreye, 1980) ${ }^{15}$.

\section{La especificidad de la acumulación en Argentina}

Argentina se encuentra entre aquellos países productores de mercancías portadoras de renta - centralmente agraria, en este caso- para el mercado mundial, cuyo desarrollo industrial general se mantuvo orientado al abastecimiento del mercado interno o regional inmediato ${ }^{16}$. La llamada nueva división internacional del trabajo no transformó esta modalidad específica, pero provocó la reducción tendencial de la renta agraria a través de sus expresiones en el movimiento global de los salarios. Simultáneamente, en el ámbito nacional, se ha ido desarrollando un proceso de centralización y concentración de capital con efectos sobre las posibilidades de compensación de la baja productividad derivada de la acotada escala del mercado nacional. Pese a las explosiones circunstanciales de la renta de la tierra agraria ${ }^{17}$ y sus formas de realización, incluida la subida relativa del salario, la escala de la producción anual de valor en Argentina comenzó a estancarse e incluso retrocedió -a través de oscilaciones, pero sostenidamente-. Ello tuvo como efecto la generación de una masa de población sobrante. La venta de la fuerza de trabajo sostenidamente por debajo de su valor se constituyó en una nueva fuente de valorización extraordinaria para los capitales que se localizan en nuestro país (Iñigo Carrera, 1998).

\footnotetext{
15 “...la emergencia de la nueva división internacional del trabajo fue originalmente guiada por la búsqueda del capital no sólo de salarios relativamente bajos, sino también de fuerzas de trabajo nacionales cuyos atributos productivos específicos incluyeran la habituación al 'trabajo intenso, colectivo y organizado' (Iñigo Carrera, 2013, p. 66) en condiciones arduas [...]” (Starosta, 2016, p. 112, traducción propia).

16 Esta especificidad está dada por la posibilidad, para los capitales que operan desde este territorio, de apropiar una parte de la renta diferencial de la tierra portada en las mercancías agrarias, en virtud de la magnitud que esta alcanza. Así, el proceso argentino de acumulación ha tenido como rasgo peculiar el hecho de que, en él, los capitales industriales que producen mercancías no portadoras de renta diferencial de la tierra lo hacen en una escala acotada que se corresponde con el tamaño del mercado interno y es sustancialmente inferior a la que rige la operación de los capitales normales que cierran su ciclo de valorización en el mercado mundial (Iñigo Carrera, 1998 y 2007).

17 En los años 1974, 1985 y 2005 y los años cercanos siguientes a cada uno de ellos.
} 
La transformación masiva de porciones de los trabajadores en sobrantes para el capital transformó su educación, su sanidad, su supervivencia una vez agotada la vida productiva, etc., en superfluas para la reproducción de aquel. A la vez, en cuanto la forma nacional de la acumulación de capital ha requerido la reproducción plena de la fuerza de trabajo ligada a la organización y el control del proceso de producción y circulación se ha pasado "a incluir en su salario individual desembolsos que antes requería la reproducción del universo de la clase obrera y que, por lo tanto, eran individualmente apropiados como un servicio de carácter universal, es decir, público" (Iñigo Carrera, 1998, p. 27). Así, las políticas de descentralización y desregulación constituyeron la forma concreta de producir y reproducir la diferenciación interna de la población obrera argentina. La provisión de educación privada, sanidad privada, seguridad privada, etc., se ha desarrollado como contracara del deterioro de su provisión pública (Iñigo Carrera, 1998, p. 27).

Como expresión de este proceso, entre las décadas del setenta y noventa se dio un proceso de descentralización del sistema educativo. Así mismo, desde mediados del siglo XX una serie de políticas de subsidios y desregulación pedagógica ha favorecido la diversificación de la oferta del sector privado y su crecimiento matricular. Esto tuvo un fuerte impacto en lo que la literatura reconoce como el fenómeno de la segmentación (Braslavsky, 1989) y la fragmentación (Tiramonti, 2004) educativas tanto entre provincias como entre el sector estatal y el privado y entre las propias escuelas dentro de cada "subsistema", generando brechas de formación y circuitos de calidad diferenciados (Tiramonti, 2004). Esta segmentación se ha constituido en una de las formas específicas de la diferenciación de la fuerza de trabajo argentina en las últimas décadas. 


\section{La lectura y escritura en la extensión de la escolaridad}

\section{La extensión de la escolaridad en la segunda mitad del siglo $X X$}

Durante la segunda mitad del siglo XX en la mayor parte del mundo se impulsaron reformas de ampliación de la obligatoriedad escolar junto con cambios académicos y curriculares en la escolaridad. Ello estuvo acompañado de una expansión progresiva de la matrícula del nivel secundario y de una extensión de los años de escolaridad promedio a nivel mundial. Estos cambios, que no se han dado de la misma forma ni en el mismo momento en todos los países o regiones, tienen como característica común la extensión de la formación general o básica integrando ofertas académicas y/o contenidos curriculares. En los países industriales clásicos, las reformas se remontan a las décadas de 1960 y 1970 y se extienden hasta fines del siglo XX (Fernández Enguita, 1986; Azevedo, 2001; Acosta, 2011). En otras regiones, la legislación que ha extendido la obligatoriedad escolar es más reciente: en su mayoría de fines de 1980, 1990 o bien los años $2000^{18}$. En el caso de la mayor parte de los países latinoamericanos, este proceso tuvo lugar durante las décadas de 1990 y 2000. Cabe aclarar, sin embargo, que a mediados del siglo XX la región también observó una expansión de la escuela secundaria, aunque mucho más moderada.

En Argentina, la extensión coincidió con una serie de reformas académicas que, entre otras características, han tendido a expandir el ciclo general y común y reducir la formación específica (Rio y Schoo, 2016). Así, a medida que una porción mayor de la población fue accediendo a los estudios secundarios, estos fueron haciéndose

18 Entre 1960 y 2010, el promedio de años de escolarización de la población mundial se vio incrementado notoria y progresivamente, aunque con importantes diferencias según las regiones. Por ejemplo, entre los países de ingresos altos de la OCDE, este promedio subió de 8.7 en 1960 a 10.9 y 12.1 en 1980 y 2000. En América Latina y el Caribe esta evolución fue de un promedio de 3.8 años, 5.3 y 7.6, respectivamente. En Asia, estos valores variaron entre 1 y 2 años promedio en 1960 a alcanzar más de 6 años, si se toma el caso de Asia oriental y el Pacífico (Cohen y Soto, 2007). También las tasas de escolarización secundaria se incrementaron a nivel mundial en las últimas décadas del siglo XX. De 1985 a 1997, estos porcentajes subieron de más del 50 a más del 60 en el caso de América Latina y el Caribe y del casi 60 a superar el 70 en los países industrializados de Asia. En el caso de los países de la OCDE, la tasa bruta de escolarización media superó el 100\% en 1997 (Carlson, 2002). 
progresivamente menos especializados. Mientras que a fines del siglo XIX y principios del XX existieron los colegios nacionales, las escuelas normales, comerciales e industriales, en 1941 se creó un ciclo intermedio compartido entre dos de esas ofertas y en los cincuenta y sesenta se constituyó un nivel medio compuesto por un ciclo básico de conocimiento general seguido por modalidades de formación especializada.

La reforma de los noventa extendió tanto la obligatoriedad como la duración de la educación básica. Cambió la estructura académica creando un nivel de Educación General Básica de nueve años, obligatorio, seguido por un nivel Polimodal de tres años no obligatorios, con orientaciones, que otorgaba título de bachiller. La última Ley de reforma educativa, de 2006, estableció la obligatoriedad del secundario y, dentro de este último, un ciclo básico de dos o tres años -dependiendo de la estructura de cada provincia- de formación común y general. Además, regimentó un proceso de transformación pedagógica del nivel secundario que se encuentra en curso. Este proceso implica, entre otros aspectos, la definición de trece orientaciones para todas las secundarias del país y, salvo en el caso de la modalidad técnica que cuenta con reglamentación particular, y en algunos casos de la modalidad artística, otorga título de "Bachiller en...", de acuerdo con la orientación elegida. También destina una mayor carga horaria a los contenidos considerados de formación general, para todos los planes de estudio del nivel secundario (Rio y Schoo, 2016).

Como advertimos en un apartado previo, el proceso de expansión del secundario en Argentina se ha dado en simultáneo con procesos de segmentación y/o fragmentación de la oferta educativa (Braslavksy, 1989; Tiramonti, 2004) y de persistencia del desgranamiento de la matrícula (Acosta, 2011). 


\section{La extensión de la escolaridad en cuanto desarrollo de las capacidades de lectura y escritura}

Los apartados precedentes nos han puesto delante de los siguientes interrogantes: ¿en qué consiste la extensión reciente de la escolaridad promedio de la población argentina en cuanto al desarrollo de sus capacidades lectoras y de escritura? ¿Cuánto hay en ella de desarrollo efectivo de atributos productivos novedosos resultantes de la automatización y computarización de los procesos de trabajo? ¿En qué medida es expresión del prolongado crecimiento de la población visiblemente sobrante para el capital?

El análisis de los resultados de pruebas de desempeño en Lengua a alumnos con trayectorias de diversa longitud en la escuela secundaria puede aportar algunos indicios sugerentes. Trabajamos sobre los resultados de las pruebas de Lengua del $\mathrm{ONE}^{19}$, desde 2007 en adelante, para alumnos en $6^{\circ}$ grado de la enseñanza primaria -lo que en la mayor parte de las jurisdicciones equivale al año de finalización de esta-, en $2^{\circ}$ o $3^{\text {er }}$ año de la enseñanza secundaria -según se trate de secundaria de 5 o 6 años de duración, respectivamente- y en el año de finalización de la enseñanza secundaria.

La selección del ONE como fuente de datos se basa en: 1) la construcción de estas pruebas como pruebas referidas a criterios a partir de 2005 , lo que permite trasladar "el foco de atención de la comparación de resultados entre alumnos o entidades hacia la descripción de lo que los alumnos saben y son capaces de hacer" (DiNIECE, 2006, pp. 3-4) y "definir varios niveles de desempeño de la capacidad" (DINIECE, 2006, p. 5); 2) el hecho de que las capacidades evaluadas en el operativo se correspondan con el de Núcleos de Aprendizajes Prioritarios y los diseños curriculares jurisdiccionales (DINIECE, 2006); 3) la posibilidad de comparar los resultados de la evaluación de capacidades cognitivas

\footnotetext{
19 El Operativo Nacional de Evaluación es ejecutado desde 1993 por la DiNIECE (DiNIECE, 2002) con una periodicidad de dos o tres años. Son evaluados los alumnos de $3^{\text {er }}$ y $6^{\circ}$ grado del nivel primario - en Lengua, Matemática, Ciencias Sociales y Ciencias Naturales- y los de $2^{\circ} / 3^{\circ}$ y año de finalización del nivel secundario —en Lengua y Matemática一 Es de carácter muestral para el nivel primario y para $2^{\circ} / 3^{\text {er }}$ año de secundario, y censal para el año de finalización del secundario.
} 
entre provincias y sectores de gestión educativa - público/privado-y, por ende, de considerar los efectos de la segmentación educativa.

\section{El contenido de la educación secundaria a partir de los aprendizajes esperados}

Los tipos de textos empleados en la evaluación de la comprensión lectora de los alumnos que finalizan el nivel primario y los de la mitad del nivel secundario no parecen presentar diferencias: en las descripciones provistas por DINIECE coinciden tanto los tipos de fuentes como la extensión y el nivel de complejidad de los textos. En la evaluación de esta capacidad entre los alumnos del último año del nivel secundario, en cambio, se utilizan cuentos de autores reconocidos, más extensos y no necesariamente de estructura canónica; entre los textos no literarios se priorizan los textos argumentativos.

Considerando textos de complejidad similar, entonces, las capacidades diferenciales mínimas de comprensión lectora que se esperan de los alumnos tras dos o tres años de educación secundaria incluyen: el reconocimiento de información textual explícita, aunque no aparezca destacada en el texto; la identificación de la secuencia de ideas o hechos que aparecen dispersas en un texto -capacidad de extraer información que a la finalización del nivel primario definía un nivel de desempeño alto-; la jerarquización de los hechos e ideas presentados en un texto, distinguiendo los principales de los periféricos; el reconocimiento y la comprensión de relaciones inferenciales de mediana complejidad; la selección de hechos o ideas principales; la relación de aspectos textuales medianamente complejos para un lector del ciclo con la propia experiencia, conocimientos e ideas, no necesariamente cercanos al mundo cotidiano; la reflexión medianamente compleja a partir de conocimiento extratextual; la capacidad de distanciarse del texto y considerarlo objetivamente al fundamentar el propio punto de vista; la identificación de elementos de la estructura de un texto con o sin uso de metalenguaje; el análisis de principios constructivos medianamente complejos de un texto literario y recursos literarios; la identificación de tipos de narradores de uso habitual. 
En cuanto a las capacidades diferenciales minimas de lectocomprensión que se esperan de los alumnos en el año de finalización de la enseñanza secundaria, más allá de los que surgen de la referida diferencia de complejidad de los textos utilizados, puede mencionarse el reconocimiento específico de la tesis que sostiene el enunciador y/o reconocimiento de diferentes voces del texto sin ayuda de la puntuación, con comprensión del uso y función de estrategias argumentativas medianamente complejas y la capacidad de reflexionar sobre las mismas (DiNIECE, 2009).

La producción de textos es evaluada únicamente durante la finalización del nivel secundario y solo en 2013. La capacidad considerada mínima consiste en escribir textos breves, coherentes en su mayor parte y legibles, con uso adecuado de elementos de cohesión variados entre párrafos y oraciones, con pocos errores en cuanto al uso del registro propio de la lengua escrita y pocos errores ortográficos o referidos a las normas de la lengua escrita (DiNIECE, 2013).

En suma, se espera que el tránsito por el nivel secundario habilite a los alumnos a identificar información dispersa no destacada e identificar la secuencia que la contiene; jerarquizar los contenidos de los textos según su centralidad, identificar las diversas voces que un texto alberga; distanciarse de su experiencia cotidiana al interpretar un texto; considerar los textos objetivamente y analizar su estructura y principios constructivos - esto es, estar en condiciones de tomar al lenguaje como objeto de conocimiento- mediante el uso de conceptos (metalenguaje); comprender y realizar inferencias y producir textos legibles y aceptablemente coherentes en un registro propio de la lengua escrita.

En los contenidos prescriptos para la educación secundaria tal como aparecen reflejados en los criterios de evaluación de Lengua, la progresión de la capacidad del sujeto de separarse del objeto concreto inmediato y comprenderlo como un caso singular de una condición más general constituye un objetivo manifiesto. 
Resulta más o menos obvia la correspondencia entre estas habilidades esperadas y la centralidad de las capacidades "heuristicas e interpretativas", de "la habilidad para interpretar la información y juzgar su significancia”, referidas por Balconi (2002, pp. 362 y 368, traducción propia). Igualmente evidente resulta que la formalización de la comunicación implicada por su modalidad computarizada supone la capacidad básica de componer textos relativamente coherentes y ajustados al registro escrito y que cierto dominio de la escritura parece la condición ineludible de un alfabetismo informático básico.

Pero existe también una correspondencia de los aprendizajes esperados con lo que Vygotsky (1983) considera el aporte específico de la educación escolar: el desarrollo de la autoconsciencia y el dominio deliberado de las habilidades. En el caso del aprendizaje de la escritura y la gramática se trata obviamente del desarrollo del control sobre el uso del lenguaje, aunque no exclusivamente. Más bien, el producto de la enseñanza de la lengua es un "nuevo uso significativo de la palabra, su utilización como un medio para la formación del concepto" (Vygotsky, 1983, p. 91), que está en la base de la transformación en el proceso intelectual cuya culminación será la capacidad de dirigir los "propios procesos mentales con la ayuda de palabras o signos" (Vygotsky, 1983, p. 91). Esto es, la capacidad para regular las propias acciones mentales, usando la palabra como medio para producir abstracciones de creciente grado de generalidad. En ese proceso de abstracción creciente, el conocimiento se hace cada vez más independiente de sus objetos inmediatos. Capacidades, todas ellas, que parecen particularmente necesarias para trabajadores cuyas habilidades, de acuerdo con Balconi (2002), están basadas en un conocimiento general y "en la capacidad de sistematizar experiencias a partir de un modelo abstracto del funcionamiento del proceso" (Balconi, 2002, p. 365, traducción propia).

\section{El contenido de la educación secundaria según los resultados obtenidos en las evaluaciones}

Si los aprendizajes mínimos prescritos al concluir la enseñanza primaria, al mediar la secundaria y al completar esta última son los referidos en el 
subapartado previo, debemos ahora dirigir la mirada a la medida en que los alumnos muestran haber realizado efectivamente esos aprendizajes en su paso por la escolaridad. Un desempeño al menos satisfactorio "en el dominio del conjunto de contenidos y capacidades cognitivas evaluadas y esperables, según los documentos curriculares jurisdiccionales y los NAP considerados como referentes" (DiNIECE, 2013, p. 9) define la pertenencia a los niveles "medio" o "alto" de desempeño. Es decir que quienes tienen un nivel de desempeño bajo no manifiestan haber llegado a dominar ese conjunto de contenidos y capacidades de la manera esperada.

Qué proporción de los alumnos evaluados en el ONE se encuentra en esta última situación en cuanto al aprendizaje de Lengua puede observarse en la Tabla 1: el tránsito por el sistema educativo no ha permitido adquirir las capacidades de lectura y escritura mínimas esperadas a entre un tercio y un cuarto -según el año considerado- de los alumnos que cursan el $6^{\circ}$ año de la educación primaria, alrededor de un cuarto de quienes promedian sus estudios secundarios y entre un quinto y un cuarto de quienes se encuentran en el año de finalización del secundario.

\begin{tabular}{|c|c|c|c|c|}
\hline \multirow{2}{*}{ Año de estudio } & $\begin{array}{c}\text { Nivel de } \\
\text { desempeño }\end{array}$ & 2007 & 2010 & 2013 \\
\hline \multirow{3}{*}{$6^{\circ}$ Primaria } & Alto & 23,2 & 22,9 & 23,3 \\
\cline { 2 - 5 } & Medio & 43,1 & 49,4 & 48,7 \\
\cline { 2 - 5 } & Bajo & 33,7 & 27,7 & 28,0 \\
\hline \multirow{2}{*}{$2^{\circ} / 3^{\circ}$ Secundaria } & Alto & 17,1 & 16,3 & 15,3 \\
\cline { 2 - 5 } & Medio & 53,7 & 59,3 & 60,3 \\
\cline { 2 - 5 } & Bajo & 29,2 & 24,4 & 24,4 \\
\hline \multirow{2}{*}{ Ú $^{\circ}$ Secundaria } & Alto & 20,2 & 20,4 & 21,3 \\
\cline { 2 - 5 } & Medio & 58,7 & 53,3 & 53,0 \\
\cline { 2 - 5 } & Bajo & 21,2 & 26,3 & 25,7 \\
\hline
\end{tabular}

Tabla 1. Alumnos según nivel de desempeño en pruebas ONE de Lengua 2007, 2010 y 2013 por año de estudio $\left(6^{\circ}\right.$ año de la enseñanza primaria, $2^{\circ} / 3^{\circ}$ y últimos años de la enseñanza secundaria)

Fuente: Informes de Resultados ONE 2007, 2010 y 2013. Recuperados de http://portales. educacion.gov.ar/diniece/2014/05/22/evaluacion-de-la-calidad-educativa-documentos 
La disparidad geográfica que presenta la incidencia de los alumnos que no demuestran haber alcanzado los contenidos mínimos esperados para el punto que transitan en su escolaridad, por su parte, deja ver que la probabilidad de alcanzarlos no es aleatoria (figuras 1 y 2 ).

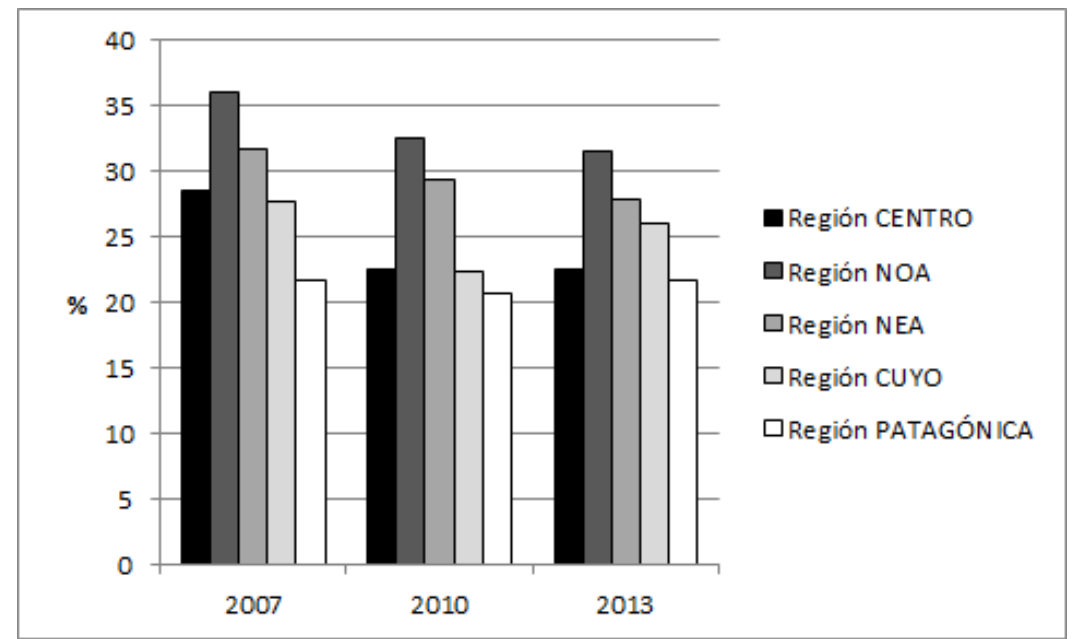

Figura 1. Porcentaje de alumnos con desempeño bajo en Lengua por región. $2^{\circ} / 3^{\circ}$ año de la enseñanza secundaria. ONE 2007-2013

Fuente: DINIECE, informes de resultados ONE 2007, 2010 y 2013

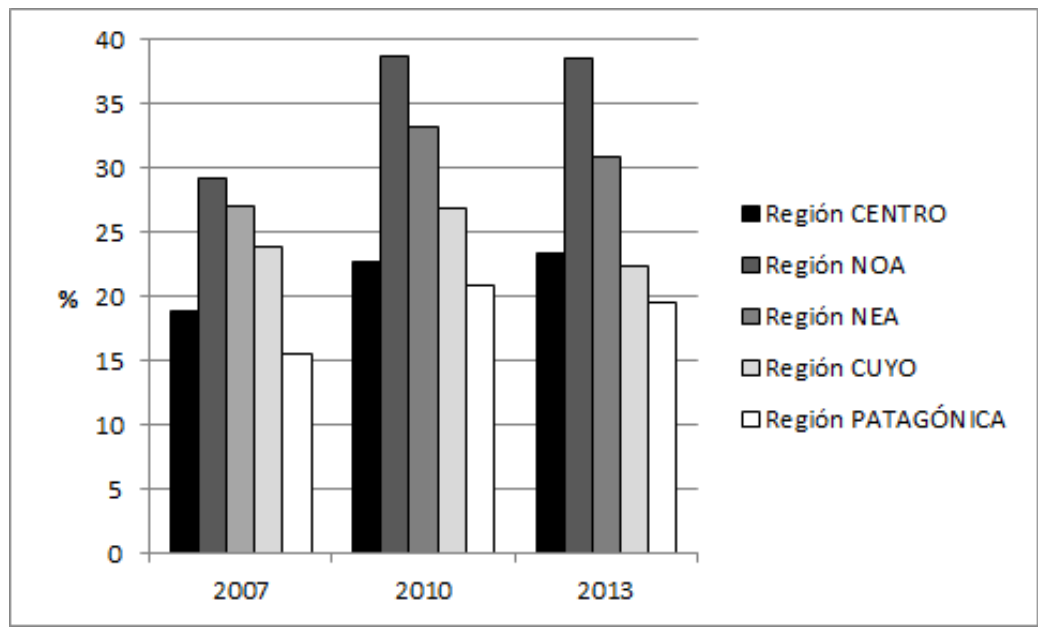

Figura 2. Porcentaje de alumnos con desempeño bajo en Lengua por región. Último año de la enseñanza secundaria. ONE 2007-2013

Fuente: DINIECE, informes de resultados ONE 2007, 2010 y 2013 
Otro tanto ocurre con el sector de las escuelas a las que los alumnos evaluados concurren (figura 3).

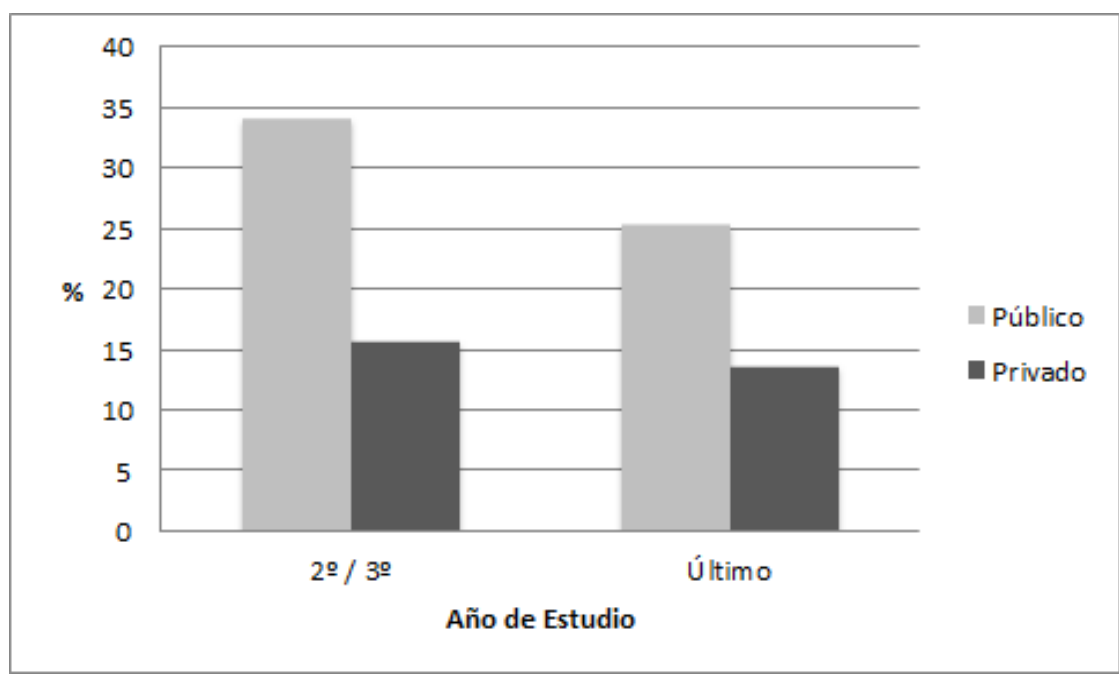

Figura 3. Porcentaje de alumnos con desempeño bajo en Lengua por sector y año de la enseñanza secundaria. ONE 2007

Fuente: DiNIECE, informes de resultados ONE 2007

Como ha sido señalado en la literatura especializada (Cervini, 2003; Dabenigno, Austral, Iñigo y Larripa, 2014), el sector de las escuelas no determina por sí mismo el desempeño de los alumnos en las pruebas, sino que, en cambio, es la mediación a través de la cual el lugar que se ocupa en el consumo social incide sobre ese desempeño. Huelga decir que el papel en el consumo social es, por su parte, la forma que realiza lo que se es para la reproducción material de la sociedad (Marx, 2001).

Lejos de resultar novedosos, estos datos son, en cambio, una expresiva reafirmación de la tesis de la segmentación educativa: también en lo que se refiere al aprendizaje de aquello que la sociedad sanciona como las capacidades minimas necesarias en el uso de la lengua castellana escrita - por la producción de las cuales el Estado nacional afirma que velará-, el tránsito por la escolaridad secundaria supone 
contenidos muy distintos según lo que cada individuo concreto sea para la reproducción social.

Hemos visto que en determinadas regiones geográficas y en la escuela pública, alrededor de un tercio de los estudiantes cursan el año intermedio de la educación secundaria sin aprender a reconocer contenidos de los textos escritos que no sean inmediatamente evidentes, jerarquizar los elementos del texto y reconocer las relaciones que guardan entre sí, relacionar los contenidos de un texto con elementos que excedan su experiencia cotidiana, tomar distancia del texto, analizarlo mediante conceptos específicos, reconocer puntos de vista diversos en él. También, que una proporción aun mayor de los alumnos que cursan el último año de la educación secundaria en las mismas regiones -así como alrededor de un cuarto de los estudiantes que lo cursan en la escuela pública- no logra, por ejemplo, identificar una tesis enunciada por escrito, reconocer voces diversas en un texto, comprender estrategias argumentativas, reflexionar sobre estas. La prolongación normativa y la extensión efectiva de los estudios de la población argentina, lejos de suponer la eliminación de la diferenciación entre porciones de la población trabajadora, han operado hasta el presente como formas de su realización.

\section{Conclusiones}

A partir del siglo XIX, la obligatoriedad escolar ha realizado la necesidad de la sociedad capitalista por la universalidad de la lectoescritura. Las transformaciones en la materialidad del trabajo durante la segunda mitad del siglo XX determinaron el crecimiento de la necesidad por trabajadores que, como minimo, puedan decodificar los mensajes enviados por los instrumentos de trabajo, evaluarlos y actuar en consecuencia; operar fácilmente con generalizaciones y adaptarlas a diversas situaciones concretas y expresarse por escrito. A nuestro entender, el proceso mundial de prolongación de la escolaridad media y la "generalización" de los contenidos del nivel secundario son expresiones de ese crecimiento. 
En el caso de Argentina, dada su especificidad histórica y la consiguiente expansión de la población obrera sobrante para el capital en los últimos cuarenta años, la extensión del secundario se realizó a través de un proceso de segmentación educativa, como modo concreto de diferenciación de la fuerza de trabajo. Los diferentes contenidos del pasaje por el nivel no se expresan solo en la diversidad de trayectorias -incluyendo el abandono temprano-, sino también en toda la variada gama de aspectos que la literatura ha señalado.

En cuanto al aprendizaje de habilidades de lectoescritura, la desigual distribución de la población relativamente sobrante sobre el territorio tiene una correspondencia evidente con la adquisición diferenciada de capacidades lectoras y escritoras por región geográfica. La diferenciación de los desempeños según otras variables que reflejan de manera más o menos directa- el lugar ocupado por los estudiantes y sus familias en la estructura social - v. gr., el sector de la escuela o el llamado "status sociocultural" de las familias, no analizado en este artículo- reafirma esa correspondencia.

En suma, la ampliación de la cobertura del nivel comprende tanto: 1) la difusión de capacidades de lectura y escritura que parecen corresponderse con las demandadas por las transformaciones recientes en los procesos de trabajo, como 2) una permanencia en el sistema educativo que no involucra el desarrollo de esas capacidades que la sociedad argentina ha sancionado como las minimas esperables al concluir la adolescencia.

\section{Referencias}

Acosta, F. (2011). Escuela secundaria y sistemas educativos modernos: análisis histórico comparado de la dinámica de configuración y expansión en países centrales y en la Argentina. Revista HISTEDBR On-line, 11(42), 3-13.

Althusser, L. (1970/1988). Ideologia y aparatos ideológicos del Estado [J. Sazbón, Trad.). Buenos Aires: Ediciones Nueva Visión. 
Azevedo, J. (2001). Continuidades y rupturas en la Enseñanza Secundaria. En C. Braslavsky (Org.), La Educación Secundaria ¿Cambio o inmutabilidad? Análisis y debate de procesos europeos y latinoamericanos contemporáneos (pp. 65-104). Buenos Aires: IIPE Unesco - Santillana.

Balconi, M. (2002). Tacitness, codification of technological knowledge and the organisation of industry. Research Policy, 31(3), 357-379.

Boudon, R. (1983). La desigualdad de oportunidades. La movilidad social en las sociedades industriales [M. Aparicio de Santander, Trad.]. Barcelona: Editorial Laia.

Bourdieu, P. (1978). Classement, déclassement, reclassement. Actes de la recherche en sciences sociales, 24(1) [puesto a disposición por Le Ministère de la jeunesse, de l'éducation nationale et de la recherche, Direction de l'enseignement supérieur, Sous-direction des bibliothèques et de la documentation]. Recuperado de http://www.persee.fr/doc/arss_0335-5322_1978_num_24_1_2613

Bourdieu, P. (1991). La distinción. Criterio y bases sociales del gusto. Madrid: Taurus.

Bourdieu, P. y Passeron, J. C. (1979/1998). La reproducción: elementos para una teoría del sistema de enseñanza. México: Distribuciones Fontamara.

Braslavsky, C. (1989). La discriminación educativa en la Argentina. Buenos Aires: Miño y Dávila Editores.

Carlson, B. (2002). Educación y mercado de trabajo en América Latina: ¿Qué dicen las cifras? [Serie Desarrollo Productivo 114]. Santiago de Chile: Naciones Unidas.

Cervini, R. (2003, febrero 10). Diferencias de resultados cognitivos y nocognitivos entre estudiantes de escuelas públicas y privadas en la educación secundaria de Argentina: un análisis multinivel. Education Policy Analysis Archives, 11(6). Recuperado de http://epaa.asu.edu/epaa/v11n6 
Chartier, A. (2002). Les modèles contradictoires de la lecture entre formation et consommation, de l'alphabétisation populaire à la lecture de masse. Cahiers de l'Association internationale des études françaises, 54, 361-380.

Chartier, A. y Hébrard, J. (2000). Saber leer y escribir: unas 'herramientas mentales' que tienen su historia. Infancia y aprendizaje, 23(89), 11-24.

Cohen, D. y Soto, M. (2007). Growth and human capital: good data, good results. Journal of economic growth, 12(1), 51-76.

Cuesta Fernández, R. (2005). Felices y escolarizados. Crítica de la escuela en la era del capitalismo. Barcelona: Octaedro - EUB.

Dabenigno, V., Austral, R., Iñigo, L. y Larripa, S. (2014). Análisis multinivel de los resultados de las pruebas PISA 2012 de Matemática en la Ciudad Autónoma de Buenos Aires [Documento de Trabajo de la UEICEE-ME-GCABA].

De Luca, R. (2004). La cobertura ideológica de la reforma educativa menemista: el Congreso Pedagógico de 1984. Razón y Revolución, 13, 93-103.

DiNIECE. (2006, septiembre). Recorrido político y técnico-pedagógico en el proceso de elaboración, justificación y validación de los criterios de evaluación. Recuperado de http://repositorio.educacion.gov.ar:8080/dspace/bitstream/ handle/123456789/109709/EVALUACION_CRITERIAL_6abril06.pdf

DiNIECE. (2009, octubre). Documentos: criterios de evaluación actualizados 2009. Recuperado de http://portales.educacion.gov.ar/diniece/2014/02/25/ criterios-de-evaluacion-actualizados-2009

DiNIECE. (2013, octubre). Documentos: criterios de evaluación actualizados 2009. Recuperado de http:/ / portales.educacion.gov.ar/diniece/2014/02/25/ criterios-de-evaluacion-one-2013

Fernández Enguita, M. (1986). Integrar o segregar. La enseñanza secundaria en los paises industrializados. Barcelona: Editorial Laia. 
Filmus, D. (1998). Hacia una nueva articulación en la relación educación trabajo. En D. Filmus (Comp.), Las transformaciones educativas. Tres desafios: democracia, desarrollo e integración (pp. 193-220). Buenos Aires: Troquel - OEI.

Foucault, M. (1976/2002). Vigilar y castigar [A. Garzón del Camino, Trad.]. México: Siglo XXI Editores.

Fröbel, F., Heinrichs, J. y Kreye, O. (1980). The New International Division of Labour: Structural Unemployment in Industrialised Countries and Industrialisation in Developing Countries. Cambridge: Cambridge University Press.

Hébrard, J. (1988). La scolarisation des savoirs élémentaires à liépoque moderne. Histoire de l'éducation [Service d'histoire de l'éducation, I.N.R.P], 38(1), 7-58.

Hirsch, M. e Iñigo, L. (2007). La formación del sistema educativo argentino: ¿producción de fuerza de trabajo vs. producción de ciudadanos? $7^{\circ}$ Conferencia presentada en el Congreso Nacional de Estudios del Trabajo, ASET, Buenos Aires.

Iñigo Carrera, J. (1993). El desarrollo del capital en acción revolucionaria consciente. Crítica de la teoria científica. Buenos Aires: CICP.

Iñigo Carrera, J. (1998). La acumulación de capital en la Argentina. Buenos Aires: CICP.

Iñigo Carrera, J. (2007). La formación económica de la sociedad argentina. Volumen 1: Renta agraria, ganancia industrial y deuda externa. 1882-2004. Buenos Aires: Imago Mundi.

Iñigo Carrera, J. (2007). Conocer el capital hoy. Usar criticamente El Capital. Buenos Aires: Imago Mundi.

Iñigo Carrera, J. (2008). El capital: razón histórica, sujeto revolucionario y conciencia, Buenos Aires: Imago Mundi.

Luria, A. (1980). Los procesos cognitivos. Análisis socio-histórico. Barcelona: Ed. Fontanella. 
Marx, K. (2001). Elementos fundamentales para la critica de la economía politica (Grundrisse) 1857-1858 [Vol. 1, 18ª Ed]. México: Siglo XXI Editores.

Marx, K. (2005). La tecnología del capital. Subsunción formal y subsunción real del proceso de trabajo al proceso de valorización [Extractos del manuscrito 1861-1863]. México: Editorial Itaca.

Marx, K. (2008). El Capital: el proceso de producción del capital. Buenos Aires: Siglo XXI Editores.

Ong, W. (1982). Oralidad y escritura. Tecnologías de la palabra. México: Fondo de Cultura Económica.

Rio, V. y Schoo, S. (2016). La educación secundaria obligatoria en los procesos de reforma contemporáneos. Políticas nacionales y acuerdos federales en torno a su universalización. En G. Ruiz (Comp.), La educación secundaria en el marco de las reformas educativas nacionales. Regulaciones federales y politicas jurisdiccionales. Buenos Aires: Eudeba. En prensa.

Starosta, G. (2016). Revisiting the New International Division of Labour Thesis. En G. Charnock y G. Starosta (Comp.), The New International Division of Labour. Global transformation and Uneven Development (pp. 79-104). Basingstoke: Palgrave Macmillan.

Tenti Fanfani, E. (1995). Títulos escolares y puestos de trabajo: elementos de teoria y análisis comparado. Revista Internacional de Educación de Adultos (CREFAL), 3(2), 35-66.

Tiramonti, G. (1993). El nivel medio del sistema educativo argentino [Serie de Documentos e Informes de Investigación $\mathrm{N}^{\circ}$ 153]. Buenos Aires: FLACSO.

Tiramonti, G. (2004). "La fragmentación educativa y los cambios en los factores de estratificación. En G. Tiramonti (Comp.), La trama de la desigualdad educativa. Mutaciones recientes en la escuela media (pp. 15-45). Buenos Aires: Manantial. 
Vigotsky, L. (1983). Pensamiento y lenguaje. Teoria del desarrollo cultural de las funciones psiquicas. Buenos Aires: La Pléyade.

\section{Cómo citar este artículo}

Iñigo, L. y Rio, V. (2017). Extensión de la escolaridad y obligatoriedad de la escuela secundaria en Argentina: el papel de la universalización de la lectura y escritura. Universitas Humanística, 83, 213-243. http://dx.doi.org/10.11144/ Javeriana.uh83.eeoe 